\title{
CÂNCER DE MAMA: O PAPEL DO ENFERMEIRO NA ASSISTÊNCIA DE ENFERMAGEM AO PACIENTE ONCOLÓGICO
}

\author{
BREAST CANCER: THE ROLE OF NURSES IN CARE FOR \\ ONCOLOGICAL PATIENT
}

\author{
Juliana da Silva ${ }^{1}$ \\ Valdeize Rego Marinho ${ }^{2}$ \\ Thaianna Cristina Oliveira Imbiriba ${ }^{3}$
}

RESUMO: INTRODUÇÃO: O câncer de mama é um dos casos mais prevalentes no mundo, acometendo geralmente, as mulheres acima dos cinquenta anos. Ainda não foi identificada uma única causa específica para o câncer de mama, visto que envolve uma combinação de eventos hormonais, genéticos, e possivelmente, ambientais que podem influenciar no desenvolvimento desta neoplasia. O prognostico de um paciente oncológico depende principalmente do diagnóstico precoce, planejamento terapêutico, tratamento e cuidados específicos o que torna o profissional enfermeiro participante ativo em todo esse processo. OBJETIVO: O presente estudo tem como objetivo identificar o papel do enfermeiro na assistência ao paciente oncológico. MATERIAIS E MÉTODOS: Trata-se de revisão integrativa, descritiva, de abordagem qualitativa. Seguiu seis etapas: estabelecimento da questão de pesquisa, busca na literatura, categorização dos estudos, avaliação dos estudos incluídos, interpretação dos resultados e apresentação da revisão pesquisadas nas bases: Scientific Electronic Library Online (SciELO), Google Acadêmico, Ministério da Saúde, Literatura LatinoAmericana e do Caribe em Ciências da Saúde (LILACS), entre 2016 a 2020, totalizando trinta e cinco artigos. RESULTADOS E DISCUSSÕES: Compreende-se que o Enfermeiro é indispensável na assistência ao paciente oncológico através da realização de consultas de Enfermagem, elaboração do planejamento terapêutico como método preventivo, aplicação de quimioterápicos, tratamento dos efeitos colaterais, monitorização dos sinais vitais, além de encaminhamentos

\footnotetext{
1 Acadêmica do curso de Bacharelado em Enfermagem, pelo Centro Universitário da Amazônia - UNAMA. E-mail: juliana2rsamira@gmail.com.

2 Acadêmica do curso de Bacharelado em Enfermagem, pelo Centro Universitário da Amazônia - UNAMA. E-mail: deizemarinhoo8@gmail.com.

3 Professora-Orientadora - Bacharelado em Enfermagem, pelo Centro Universitário da Amazônia UNAMA. Especialista em Saúde Pública com Ênfase em Saúde da Família. E-mail: thaiana.imbiriba@hotmail.com.
} 
para outros setores e equipe multiprofissional. A prevenção primária relaciona-se ao controle dos fatores de riscos, principalmente no que se refere ao estilo de vida e ao diagnóstico precoce. Essa detecção precoce é o início imediato do tratamento do câncer de mama o que de fato reduz a taxa de mortalidade da neoplasia. CONSIDERAÇÕES FINAIS: O enfermeiro tem o papel de educador e coordenador de ações de prevenção, diagnóstico e tratamento de mulheres com câncer de mama, tendo competência para oferecer assistência à paciente e habilidade para intervenções no combate a esse câncer, através de palestras, campanhas para exames, orientações de prevenção e tratamento, disseminando a importância do diagnóstico precoce, visando a redução de novos casos e mortalidade feminina.

Palavras-Chave: Neoplasia da Mama. Diagnóstico Precoce.Cuidados de Enfermagem.

ABSTRACT: INTRODUCTION: Breast cancer is one of the most prevalent cases in the world, generally affecting women over fifty. A single specific cause for breast cancer has not yet been identified, as it involves a combination of hormonal, genetic, and possibly environmental events that can lead to the development of this neoplasm. The prognosis of a cancer patient depends mainly on early diagnosis, therapeutic planning, treatment and specific care, which makes the professional nurse an active participant in this entire process. OBJECTIVE: This study aims to identify the role of nurses in cancer patient care. MATERIALS AND METHODS: This is an integrative, descriptive review with a qualitative approach. There were six steps: establishment of the research topic, literature search, categorization of studies, evaluation of included studies, interpretation of results and presentation of researched reviews in the following databases: Scientific Electronic Library Online (SciELO), Academic Google, Ministry of Health, Latin American Literature and Caribbean in Health Sciences (LILACS), from 2016 to 2020 , totaling thirty-five articles. RESULTS AND DISCUSSION: It is understood that the Nurse is essential in the care of cancer patients through nursing consultations, planning of therapeutic planning as a preventive method, application of chemotherapy, treatment of effects, monitoring of vital signs, in addition to referrals to other sectors and a multidisciplinary team. Primary prevention is related to the control of risk factors, mainly not related to lifestyle and early diagnosis. This early detection and prompt initiation of breast cancer treatment is what actually reduces the cancer mortality rate. FINAL CONSIDERATIONS: The nurse has the role of educator and coordinator of prevention, diagnosis and treatment actions for women with breast cancer, having the competence to offer patient care and the ability to treat this cancer through lectures, campaigns for exams, prevention and treatment guidelines, disseminating the importance of early diagnosis, aiming to reduce new cases and female mortality.

Keywords: Breast Neoplasm. Early diagnosis. Nursing care. 


\section{INTRODUÇÃO}

Feitosa et al. (2018) relatam que o câncer é uma doença com localizações e aspectos clínicos múltiplos, podendo ser diagnosticado em diversos estágios. Caracteriza-se por sua complexidade, tornando, com isso, necessário estudo contínuo e profissional especializado.

Para os autores acima citado, não há causa única específica para o câncer de mama; em vez disso, uma combinação de eventos hormonais, genéticos e, possivelmente, ambientais pode contribuir para seu desenvolvimento.

O Instituto Nacional de Câncer - INCA (202I) apresenta como estimativa para o câncer de mama no Brasil, para o triênio 2020/2022 que sejam diagnosticados 66.280 novos casos dessa neoplasia, com um risco estimado de 61,6I casos a cada ioo mil mulheres.

Em análise mundial, o câncer de mama é o mais incidente entre as mulheres, conforme relata o INCA (2021), sendo que em 2018 houveram 2.I milhões de casos novos mundialmente. No Brasil, em 2020, foram detectados 66.280 novos casos desta neoplasia.

De acordo com Santos e Chubaci (2017), com o uso crescente da mamografia, as mulheres estão procurando tratamento em um estágio mais precoce da doença. Essas mulheres podem não apresentar sintomas e nenhuma nodosidade palpável, mas as lesões anormais são detectadas à mamografia. Infelizmente, muitas mulheres com doenças avançadas procuram o tratamento inicial somente depois de ignorar os sintomas.

Para Derenzo et al. (2017), quando os sinais se tornam mais evidentes, caracteriza-se os estágios tardios do câncer de mama, prejudicando a recuperação completa da paciente.

Conforme Oliveira et al. (2017), o prognóstico de um paciente oncológico, além das condições inerentes ao tipo de câncer, depende fundamentalmente do diagnóstico precoce, planejamento terapêutico correto, tratamento e cuidados efetivos e eficazes na assistência. Nesse sentido, o enfermeiro oncologista é participante ativo em todo esse processo, geralmente prolongado e debilitante.

De acordo com Alves e Fernandes (2017), a grande maioria das mulheres teme o diagnóstico de câncer de mama, provavelmente devido a alta incidência, além de ser um risco genético. Outro ponto que fortalece esse temor, são efeitos psicológicos derivados da possível retirada das mamas, como forma de tratar o câncer. Por ser o tipo de câncer mais 
comum entre as mulheres, a quantidade de casos novos aumenta a cada ano, ampliando a sua incidência em todo mundo.

Neste contexto, Feitosa et al. (2018) discorrem que o papel do enfermeiro é fundamental no processo de prevenção visto que são estes profissionais que orientam os pacientes na prevenção primária relacionado aos melhores hábitos de vida.

Oliveira et al. (2017) ressaltam que como educador, o enfermeiro possui capacitação e autonomia na realização de palestras, campanhas, além da solicitação de exames, e aplicar a medicação dentro dos protocolos existentes no atendimento a paciente com esta patologia. Através da atuação do enfermeiro é essencial no diagnóstico precoce do câncer de mama, reduzindo assim os riscos de uma descoberta tardia.

Além disso, conforme Pereira et al. (2018), é importante a divulgação do conhecimento científico na área, integrando ensino-serviço e contribuindo para a melhora da qualidade do cuidado de enfermagem.

Diante disso, houve a necessidade de identificar o papel do enfermeiro na assistência ao paciente oncológico, propondo uma avaliação dos principais fatores que afetam a qualidade da assistência buscando a qualidade no atendimento e satisfação do usuário.

Neste contexto, ampliar os conhecimentos sobre o atendimento de enfermagem a pacientes com câncer de mama é de grande relevância para que se possa realizar uma abordagem humanizada e holística deste paciente, podendo assim, avaliar e assistir de forma adequada e singularizada, auxiliando na melhora da qualidade de vida desta pessoa.

Com embasamento dos fatos citados esse estudo pode servir de base para todos os profissionais da área de saúde ou que trabalhem diretamente no setor oncológico com o objetivo de avaliar o papel do enfermeiro na assistência ao paciente oncológico, bem como quais as dificuldades na sua implementação

\section{FUNDAMENTAÇÃO TEÓRICA}

\section{I Câncer de Mama}

Segundo Smeltzer e Bare (2017), os cânceres de mama ocorrem em qualquer região na mama, porém a maioria é encontrada no quadrante superior externo, onde se localiza a maior parte do tecido mamário. Em geral, as lesões são indolores, ao invés de dolorosas; 
fixas, ao invés de móveis; e induradas com bordas irregulares, em vez de encapsuladas e lisas.

Bezerra et al. (2019) descrevem que o câncer de mama geralmente inicia nas células do epitélio que reveste a camada interna do ducto mamário. É raro o câncer de mama iniciar em outros tecidos, tais como o adiposo e o fibroso da mama. Os autores salientam que esta neoplasia decorre de alterações genéticas, todavia é possível ocorrer devido a hereditariedade, que corresponde cerca de $5 \%$ a I0\% dos casos.

De acordo com Rezende et al. (2018), o câncer inicia nas células, para em seguida, espalhar-se pelo órgão. As células normais da mama e de outras partes do corpo crescem e se dividem em novas células. Quando as células antigas não podem mais se reproduzirem, elas morrem, dando vez às novas células. Este acúmulo de células que não estão de forma necessária, frequentemente se transformam em uma massa de tecido.

Zuca-Mathes (2018) expõe que tumores na mama podem ser benigno (não cancerosos) ou maligno (canceroso). Os tumores benignos, geralmente não são prejudiciais raramente invadem tecidos sadios, não se espalhando para outras partes do corpo, eles podem ser removidos e geralmente não crescem.

Neto (2019) narra que os tumores malignos, podem invadir os órgãos e tecidos próximos, espalhando-se por outras partes do corpo, geralmente podendo ser removidos, mas às vezes voltam a crescer. As células também podem se espalhar para os gânglios linfáticos, parte do sistema imunológico do corpo, e para os pulmões, fígado, ossos e cérebro; o que se chama de câncer de mama metastático.

Segundo Alves e Fernandes (2017), o carcinoma in situ de mama está sendo detectado com maior frequência com o uso disseminado da mamografia de triagem. Essa doença caracteriza-se pela proliferação das células malignas dentro dos duetos e lóbulos, sem invasão para o tecido circunvizinho; portanto, é uma forma de câncer não-invasiva e é considerado câncer de mama em estágio o. Existem dois tipos de carcinoma in situ: ductal e lobular.

O carcinoma ductal in situ é o mais comum dentre os dois tipos, é dividido histologicamente em dois subtipos principais (comedão e não-comedão), mas existem muitas formas diferentes do carcinoma ductalin situ - DCIS nãocomedão. Como o DCIS apresenta a capacidade de progredir para câncer invasivo, 
o tratamento mais tradicional é a mastectomia total ou simples (apenas a remoção da mama), com uma taxa de cura de 98 a 99\%. (SMETLZER; BARE, 2017, p. 154I).

Neto (2019) descreve que o carcinoma lobular in situ caracteriza-se por proliferação das células dentro dos lóbulos da mama. Em geral, esse tipo é um achado incidental descoberto na avaliação patológica de uma biópsia de mama para uma alteração mamária percebida durante o exame físico ou em uma mamografia de triagem. Ele está comumente associado a doença multicêntrica e raramente ao câncer invasivo.

Rezende et al. (2018) referem que o carcinoma invasivo podem ser classificados em carcinoma ductal infiltrativo, carcinoma lobular infiltrativo, carcinoma medular, câncer mucinoso, câncer ductal tubular, carcinoma inflamatório e a doença de Paget.

Os autores supracitados discorrem que os carcinomas ductais infiltrativos constituem o tipo histológico mais comum de câncer de mama e contribuem com $75 \%$ de todos os cânceres de mama. Esses tumores são notáveis por causa de sua induração à palpação. Em geral, eles enviam metástases para os linfonodos axilares. O prognóstico é pior em comparação a outros ti-pos de câncer.

Segundo Bezzerra et al. (2019), no carcinoma lobular invasor o achado típico é o de uma área mal definida, infiltrada, espessada na mama, com pouca expressão mamográfica. Costuma ser multicêntrico e com maior tendência a bilateralidade. Pode se apresentar mais raramente como a doença de Paget do mamilo - manifestada como escoriações no mamilo; e como o câncer de mama inflamatório - caracterizada por uma constelação de calor, rubor e edema.

De acordo com Alves e Fernandes (2017), o carcinoma medular constitui aproximadamente 6\% dos cânceres de mama e cresce em uma cápsula dentro de um dueto. Esse tipo de tumor pode ficar grande, porém o prognóstico é favorável.

Os autores supracitados narram que o câncer mucinoso contribui com cerca de 3\% dos cânceres de mama. Como produtor de mucina, ele também exibe crescimento lento e, assim, apresenta prognóstico mais favorável do que muitos outros tipos.

O câncer ductal tubular, conforme Zuca-Mathes (2018), contribui apenas com 2\% dos cânceres. Como as metástases axilares são incomuns com essa histologia, o prognóstico geralmente é excelente. 
De acordo com Smeltzer e Bare (2017, p. 291), “o carcinoma inflamatório é um raro tipo de câncer de mama ( a 2\%) com sintomas diferentes daqueles de outros cânceres de mama”. O tumor localizado é doloroso e sensível, e a pele sobre ele mostra-se avermelhada e mosqueada. A mama fica anormalmente firme e aumentada, ocasionando edema e a retração do mamilo.

Segundo Neto (2019), a doença de Paget da mama contribui com I\% dos casos de câncer de mama diagnosticados. É considerada uma lesão descamativa e a queimação e o prurido ao redor do complexo mamilo-aréola são sintomas frequentes. A neoplasia é ductal e pode ser in situ, isoladamente ou ter também células cancerosas invasivas. Com frequência, uma massa tumoral não pode ser palpada sob o mamilo onde surge essa doença.

\subsection{Programa Câncer de Mama}

O Ministério da Saúde (MS) é o órgão responsável pelo investimento das ações de prevenção do câncer. De acordo com Alves e Fernandes (2017), os principais objetivos de programas de controle da doença são a redução de sua incidência e o aumento da cura dos

diferentes tipos de câncer. $O$ investimento nas ações primárias reduziria os gastos tão elevados do governo no tratamento da doença.

Ohl et al. (2016) explanam que o câncer de mama se desenvolve devido a vários fatores, tanto biológicos como ambientais, destacando àqueles referentes à idade, aspectos endócrinos e genéticos. Neste contexto, a prevenção primária desta neoplasia relaciona-se ao controle dos fatores de riscos, principalmente no que se refere ao estilo de vida e ao diagnóstico precoce, rastreando as mulheres com sinais e sintomas da doença.

Segundo Migowski et al. (2018), foi a partir da criação do Sistema Único de Saúde (SUS), que o governo passou a desenvolver ações para a detecção precoce do câncer de mama, inicialmente pelo Programa Viva Mulher, de 1996 a 2003, recomendando o rastreamento mensal com autoexame e exame clínico anual, realizados por médicos e enfermeiros, em mulheres a partir dos 40 anos, reservando a mamografia para confirmação diagnóstica. 
Os autores supracitados expõem que o Ministério da Saúde, em parceria com todos profissionais, buscaram construir um documento que apresentasse maior subsídios para a deteç̧ão precoce dessa neoplasia. Foi através do Instituto Nacional

de Câncer - INCA, da Área Técnica da Saúde da Mulher e com o apoio de sociedades médicas, o Ministério da Saúde organizou a Oficina de Trabalho para Elaboração de Recomendações ao Programa Nacional de Controle do Câncer de Mama, realizada em novembro de 2003.

Teixeira e Araújo Neto (2020) ressaltam que nessa oficina foi elaborado um documento definindo as diretrizes nacionais para a detecção precoce do câncer de mama, incluindo a prevenção primária, detecção precoce, diagnóstico, tratamento e cuidados paliativos. O rastreamento mamográfico tornou-se uma estratégia de saúde pública, sendo reforçado em 2006, na publicação do Pacto pela Vida, incluindo diretrizes operacionais que ampliavam a cobertura deste rastreamento para 60\% das mulheres.

Gonçalves et al. (2016) narram que em 2005 foi lançado a Política Nacional da Atenção Oncológica e o Plano de Ação para o Controle do Câncer de Útero e de Mama. O Plano de Ação teve estratégias voltadas para a prevenção, ampliação de oferta de serviços para detecção em estágios iniciais, tratamento e reabilitação, com o intuito de reduzir a ocorrência, incidência e mortalidade por câncer de mama, além de prevenir as repercussões físicas, psíquicas e sociais da neoplasia. Observa-se que o Plano de Ação estava voltado principalmente em ampliar o rastreamento de novos casos, prevenindo que as mulheres chegassem a estágios mais avançados da neoplasia, através do diagnóstico precoce, além de oferecer maior qualidade nos exames realizados.

Neto (2019) elenca que as diretrizes e estratégias do Programa estabeleceram formar uma rede nacional integrada, baseada em um núcleo geopolítico que gerencia as ações em cada município, permitindo ampliar o acesso da mulher aos serviços de saúde. Outro ponto, foi capacitar os recursos humanos e motivar as mulheres nos cuidados de saúde, fortalecendo e aumentando a eficiência da rede formada para o controle do câncer.

Segundo Gonçalves et al. (2016), em 2008, o Ministério da Saúde instituiu o Sistema de Informação do Câncer de Mama - SISMAMA, um sistema de informação que deve fornecer dados informatizados dos procedimentos relacionados ao rastreamento e a 
confirmação diagnóstica do câncer de mama, gerando dados que subsidiam o monitoramento e a avaliação por meio de relatórios. Sua primeira versão foi desenvolvida pelo DATASUS, tendo como parceiro o INCA.

O Ministério da Saúde continuou a desenvolver novas estratégias para o controle do câncer de mama. Gonçalves et al. (2016) relatam sobre o Encontro Nacional sobre Rastreamento do Câncer de Mama, em 2009, reunindo especialistas nacionais e internacionais, além de autoridade, profissionais da saúde, sociedades cientificas e representantes da sociedade civil organizada. Também neste mesmo ano, apresentou-se os Parâmetros Técnicos para o Rastreamento do Câncer de Mama, subsidiando o planejamento e regulação das ações para rastrear esta neoplasia.

Os autores supracitados aludem sobre o Plano Nacional de Fortalecimento da Rede de Prevenção, Diagnóstico e Tratamento do Câncer, em 2olı, intensificando as ações de controle nos estados e municípios, através de investimentos técnicos e financeiros. $O$ Programa de Mamografia Móvel, em 2012, instituído através da Portaria MS/SAS no 1.228, facilitando o acesso a mamografia das residentes dos municípios brasileiros. Em 2013, através da Portaria MS/GM № 874, de 16 de maio de 2013, instituiu a Política Nacional para a Prevenção e Controle do Câncer na Rede de Atenção à Saúde das Pessoas com Doenças Crônicas.

Além destas estratégias, o Ministério da Saúde, segundo Gonçalves et al. (2016), instituiu em 2013, O Sistema de Informação de Câncer - SICAN, permitindo o monitoramento das ações referentes a deteç̧ão precoce, confirmação diagnóstica e início do tratamento de neoplasias malignas. Em 2014, foi instituído o Serviço de Referência para Diagnóstico de Câncer de Mama (SDM), oferecendo incentivos financeiros de custeio e de investimento para ser implantados em todo território nacional.

Teixeira et al. (2017) discorrem que em 2015, o Ministério da Saúde aprovou as Diretrizes para Detecção Precoce do Câncer de Mama, estabelecendo ações que se baseiam em evidências científicas, tornando-as mais efetivas e oferecendo menor dano à saúde da população. 


\subsection{A Importância do Enfermeiro no Tratamento ao Paciente Oncológico}

Silva et al. (2020) comentam que o tratamento do câncer de mama pode incluir uma série de combinações entre cirurgia, radiação, quimioterapia e terapia hormonal. Tudo depende do estágio e do tipo de câncer, da idade e das condições das mulheres e dos efeitos desfigurantes da cirurgia.

Segundo Alves e Fernandes (2017), com a finalidade de tratar o câncer de mama nos seus mais variados tipos histológicos, há três modalidades básicas terapêuticas: a cirurgia e a radioterapia, para o tratamento locorregional, e a quimioterapia e a hormonioterapia inseridas no tratamento sistêmico. Recentemente, foram inclusos no tratamento do câncer de mama os anticorpos monoclonais que atingem o antígeno c-erb-2.

Para Smeltzer e Bare (2017), o tratamento cirúrgico é a modalidade mais antiga, principalmente de tumores sólidos. Consiste em um tratamento que compreende a retirada do tumor com certa margem de segurança. No câncer de mama, a indicação cirúrgica vai depender do estadiamento clínico e do tipo histológico, podendo ser conservadora a ressecção do segmento da mama (engloba a setorectomia, a tumorectomia alargada e a quadrantectomia), com retirada de gânglios axilares ou não conservadora (mastectomia),

Conforme Bezzerra et al. (2019), a radioterapia tem desempenhado importante papel no controle do câncer de mama, especialmente para pacientes que foram submetidos a cirurgia conservadora, reduzindo a recidiva local de 35 para $8 \%$. As pacientes que foram submetidas a mastectomia radical modificada também são indicadas para a radioterapia, quando apresentam fatores de mau prognóstico local, como tumor maior que $4 \mathrm{~cm}$, invasão do tecido adiposo, invasão de bainhas nervosas e vasculares, manifestações epidérmicas préoperatórias e axila extensamente comprometida.

De acordo com Zuca-Matthes (2018), a quimioterapia, a terceira modalidade do tratamento, é amplamente utilizada devido à estratégia de tratar micrometástases para o controle da doença. Representa a unidade de tratamento sistêmico que pode ser classificada em adjuvante, neoadjuvante e paliativa. A adjuvante antecede os tratamentos locorregionais, cirurgia ou radioterapia. $\mathrm{Na}$ neoadjuvante, a terapia locorregional é feita previamente. Já, na 
quimioterapia paliativa, o objetivo é reduzir as células tumorais, consequentemente, diminuindo os sinais e sintomas relacionados à doença.

Silva et al. (2020) relatam que a quimioterapia e a hormonioterapia são tratamentos adjuvantes do câncer de mama que têm importante papel no controle da doença a distância, Visto que o tratamento locorregional não afeta substancialmente a sobrevida, entretanto o controle da doença apresenta melhores resultados.

Sousa et al. (2019) ressaltam que a importância de realizar o tratamento de câncer de mama em tempo oportuno possibilita a redução da mortalidade por esta neoplasia. Porém, os autores detectaram que existe um atraso de mais de 60 dias entre o diagnóstico e o início do tratamento, tendo como consequência um grande percentual de mulheres que iniciam o tratamento em estágio mais avançado da doença.

Segundo Teixeira e Araújo Neto (2020), a detecção precoce e o início imediato do tratamento do câncer amplia a taxa de cura das mulheres com câncer de mama, reduzindo a taxa de mortalidade da neoplasia.

Ferrari et al. (2018) narram que o enfermeiro é o profissional que acompanha diretamente todo o processo, que se inicia durante a orientação para a realização do autoexame de mama, e outros exames que possam auxiliar na prevenção desse tipo de câncer. São estes profissionais que orientam durante o tratamento quimioterápico e radioterápico, discorrendo sobre seus efeitos adversos, esclarecendo dúvidas e instruindo a forma correta do autocuidado. Os autores ressaltam a importância do enfermeiro na realização da educação em saúde para as mulheres que estão em tratamento contra o câncer de mama.

Conforme Melo et al. (2017), o enfermeiro da Unidade Básica de Saúde - UBS é o profissional da equipe multidisciplinar que realiza o rastreamento relevante que estes profissionais sejam capacitados continuamente sobre os fatores de risco, métodos de prevenção, e rastreamento de casos desse tipo de câncer na comunidade onde a UBS está inserida.

Rodrigues et al. (2020) salientam que a sistematização de assistência de enfermagem tem uma grande importância no processo de educação e sensibilização da população na promoção do autocuidado para detectar precocemente o câncer de mama e reduzir a quantidade de casos. Os autores destacam que os enfermeiros devem atuar em todos os 
níveis de atenção, primária, secundária ou terciária, visto que tem o papel fundamental no processo educativo em saúde.

De acordo com Coberlini et al. (2019), a mastectomia é a forma mais comum de tratamento do câncer de mama, em que ocorre a remoção de um ou dos dois seios da mulher, o que interfere no sentimento e na feminilidade da mulher. Nesse ponto, a atuação do enfermeiro deve ser eficaz, não somente nos cuidados de saúde, mas também na orientação dos efeitos causados pelos tratamentos, na atenção dada a mulher, e principalmente, na escuta de sua percepção sobre a forma como o tratamento está modificando a vida dela.

Silveira et al. (2021) mencionam que o enfermeiro, além do preparo do autocuidado, também deve realizar a promoção em saúde, tendo a imensa responsabilidade de realizar ações, criando estratégias, e utilizando tecnologias para rastrear e realizar o diagnóstico precoce das mulheres com câncer de mama. E durante o tratamento, deve orientar sobre a higiene do local em que foi retirado a mama, ajudando no fortalecimento do suporte para a paciente após a cirurgia.

Muller et al. (2018) referem que a enfermagem se tornou um alicerce para mulher com câncer de mama, visto que estão ao seu lado durante do diagnóstico à reabilitação e ressocialização dessas mulheres. Os autores evidenciam que os cuidados de enfermagem durante o tratamento de câncer de mama visam a conservação da capacidade funcional da mulher, especialmente durante o tratamento quimioterápico, controlando os sinais e sintomas que podem trazer incapacidade funcional para a mulher.

Belfort et al. (2019) salientam que o enfermeiro tem um papel muito importante no rastreio das mulheres com câncer de mama, e por isso, é muito importante que esses profissionais recebam capacitação e educação continuada, de maneira que possam dar um atendimento de qualidade para as mulheres, sem esquecer dos enfermeiros que realizam o atendimento da mulher durante o tratamento.

Souza et al. (2020) evidenciam que a atuação do enfermeiro em oncologia está além do cuidado técnico, visto que os novos tratamentos contra o câncer trouxe a necessidade de um trabalho multidisciplinar, em que o profissional deve realizar o atendimento técnico, mas também dar o suporte psicológico para as pacientes. Os autores discorrem que o enfermeiro pode oferecer informações sobre os efeitos e benefícios do uso de terapias 
complementares ao tratamento oncológico, visto que esse profissional tem um contato direto e prolongado com a paciente, durante o tratamento e a reabilitação da doença, oportunizando um cuidado mais centrado na mulher e nas suas necessidades.

Custódio (2019) reforça que a atuação do enfermeiro no tratamento do câncer de mama na coordenação do cuidado busca regular o plano de tratamento, evitando que a paciente se perca na linha de cuidado, realizando procedimentos desnecessários, e assim evitando que a mulher tenha sequelas devido ao tratamento recebido.

Inácio e Venson (2020) enfatizam que durante o tratamento quimioterápico, as mulheres apresentam muitas queixas, e que o enfermeiro pode orientar e realizar educação em saúde para evitar que aumentem as sequelas do tratamento na mulher, especialmente se a mesma passou pela mastectomia. A atenção do enfermeiro durante todo o processo de tratamento e reabilitação auxilia na sensação de segurança da mulher, e consequentemente, intervindo na melhoria da adesão ao tratamento.

Carreiro et al. (2017) acentuam que o profissional de enfermagem, no processo de reabilitação da mulher, tem o papel de ofertar informações referente aos cuidados pós-

operatório, orientações sobre as etapas de recuperação, cuidados com o local da cirurgia, além de informar sobre as próximas etapas do tratamento, como a radioterapia e a quimioterapia. O enfermeiro também deverá buscar informações de terapias que possam melhorar a qualidade de vida física e psicológica da paciente. Essas ações podem ser realizadas a partir da consulta de enfermagem, consistindo em uma prática assistencial que visa a promoção e reabilitação a saúde da paciente com câncer de mama.

\section{MÉTODOS}

A atual pesquisa trata-se de uma revisão integrativa de literatura, descritiva, de abordagem qualitativa que busca investigar e analisar o papel do Enfermeiro na Assistência ao paciente Oncológico.

Sousa et al. (2017) relatam que a revisão integrativa de literatura é um método muito utilizado na construção de publicações científicas em enfermagem, visto que possibilita resumir o passado da literatura empírica ou teórica, fornecendo uma compreensão mais ampla do objeto de estudo. 
Segundo Correa (2018), a pesquisa descritiva tem o intuito de descrever com detalhes um objeto de estudo, seja ele uma ação, experimento ou estático. O conhecimento prévio sobre o objeto de estudo e de sua contextualização é muito importante, visto que a descrição se baseia em comparações que irão definir o objeto descrito.

A pesquisa bibliográfica, conforme Severino (2017), realizada em livros e documentos é o primeiro passo para a construção de uma pesquisa, pois ela irá dar o suporte para a construção de um novo conhecimento, e servirá de base para o aprofundamento dos temas.

Para Lakatos e Marconi (2017), este tipo de pesquisa, considerada de fontes secundárias, abarca toda bibliografia que existe em relação a um tema, sejam estas impressas, orais ou audiovisuais. Desta forma, o pesquisador tem contato direto com tudo o que já foi escrito, dito ou filmado sobre o seu objeto de estudo.

Estudos com métodos qualitativos, de acordo com Dias (2017), se refere a técnica que busca entender as dinâmicas do objeto de estudo, enfocando as características qualitativas do mesmo, sem buscar relações a números e quantidade.

A revisão da literatura realizada antecipadamente possibilitou aos pesquisadores a aquisição de conhecimentos, que conduziu o percurso na procura de resolução de um problema. $\mathrm{O}$ acervo de informações em meios eletrônicos é um importante progresso para os pesquisadores, visto que esse acesso proporciona uma atualização frequente.

Para a coleta de dados foi realizado um levantamento de informações relacionados ao objeto de estudo através de livros e também artigos em bases de dados: Scientific Electronic Library Online (SciELO), Google Acadêmico, Ministério da Saúde, Literatura LatinoAmericana e do Caribe em Ciências da Saúde (LILACS). A estratégia utilizada para localizar os artigos foi por intermédio da combinação dos seguintes descritores e suas combinações na língua portuguesa, "Câncer de Mama”, "Enfermeiro”, "Assistência”.

Os critérios de inclusão adotados para orientar a busca e seleção das publicações foram: artigos publicados em periódicos científicos nacionais revisados por pares que abordem a temática constante no banco de dados Scientific Electronic Library Online (SciELO), Google Acadêmico, Ministério da Saúde, Literatura LatinoAmericana e do Caribe em Ciências da Saúde (LILACS), publicados entre o período de 2016 a 202I, ou seja, 
nos últimos 05 anos; artigos na sua totalidade que apresentassem a temática referente a revisão integrativa e artigos publicados e indexados nos referidos bancos de dados nos últimos cinco anos.

Os critérios de exclusão foram: As publicações que não se encontravam disponíveis em texto completo; as publicações que apresentavam disponibilidade de texto completo, mas cujo link apresentava erro mediante a tentativa de acessá-lo e os artigos fora do período determinado 2016 a 2021.

Os dados da pesquisa foram obtidos através de revisão integrativa, com base da seguinte questão norteadora: Qual o papel do enfermeiro na assistência ao paciente oncológico?

A coleta de dados dos artigos se deu entre os meses de março a julho de 2021, e a apresentação e discussão dos resultados encontrados foi feita de forma descritiva, possibilitando ao leitor a avaliação da aplicabilidade da revisão integrativa elaborada, de forma a atingir o objetivo desse método, ou seja, avaliar o papel do enfermeiro na assistência ao paciente oncológico.

\section{RESULTADOS E DISCUSSÃO}

$\mathrm{Na}$ presente revisão integrativa foram analisados trinta e cinco publicações científicas, que estavam de acordo com os critérios de inclusão pré-estabelecidos. Após a análise, foram criadas 3 categorias capazes de sinalizar as evidências cientificas disponíveis nas literaturas sobre o Papel do Enfermeiro na Assistência ao Paciente Oncológico, conforme pode ser visto nos tópicos anteriores.

\section{CONSIDERAÇÕES FINAIS}

Observou-se através do estudo que, o câncer de mama apresenta taxa elevada de óbitos, daí, a necessidade de ampliação de políticas públicas, incentivando as pesquisas com o intuito de descobrir formas de inibir o avanço da doença, bem como investir em ações educativas. Essas ações educativas visam identificar nas mulheres os fatores de risco de proliferação da doença, bem como formas de prevenção e principalmente enfatizar a 
importância da realização do exame preventivo com objetivo de detectar as alterações das mamas.

Os dados obtidos no estudo possibilitaram o alcance dos objetivos pré-estabelecidos, oportunizando identificar o papel do enfermeiro na assistência ao paciente oncológico. Nesse sentido, o enfermeiro é participante ativo em todo esse processo.

Outro fator importante e essencial foi constatar a importância do prognostico deum paciente oncológico, além das condições inerentes ao tipo de câncer, diagnóstico precoce, planejamento terapêutico, tratamento e cuidados efetivos e eficazes na assistência

No processo de pesquisa, este estudo permitiu ampliar o conhecimento sobre o câncer de mama, e seus impactos na vida das mulheres, assim como desvelar a relevância do enfermeiro na prevenção de novos casos, através do ensino do autoexame e do direcionamento para exames específicos, além da estimulação ao autocuidado e a participação da mulher no seu tratamento. É importante frisar a capacitação do mesmo referente ao conhecimento científico contribuindo para a assistência de qualidade ao paciente oncológico.

Portanto o Enfermeiro tem o papel de educador e coordenador de ações de prevenção, diagnóstico e tratamento de mulheres com câncer de mama, tendo competência para oferecer assistência à paciente e habilidade para intervenções no combate a esse câncer, através de palestras, campanhas para exames, orientações de prevenção e tratamento, disseminando a importância do diagnóstico precoce, visando a redução de novos casos e mortalidade feminina.

Com base na literatura analisada espera-se realizar contribuições para os profissionais da Enfermagem, acadêmicos, comunidade geral sobre a temática, sendo assim, fonte de informação para a realização de novas pesquisas sobre o tema abordado.

\section{REFERÊNCIAS}

ALVES, Pricilla Cândido; FERNANDES, Ana Fátima C. Câncer de mama. São Paulo: Novas Edições Acadêmicas, 2017.

BELFORT, Lucas Rafael Monteiro; LIMA, Kedma de Magalhães; DUTRA, Luciana Paula Fernandes; NEGRO-DELlACQUA, Melissa; MARTINS, Victor Hugo da Silva; 
MACEDO, Leyliane Jannice de Andrade. O papel do enfermeiro no diagnóstico precoce do câncer de mama na atenção primária. Research, Society and Development, v. 8, n. 5, p. oI13, 2019.

BEZZERRA, Diego Aragão de; DOURADO, Guilherme Peixoto; PINTO, Vicente de Paulo Teixeira; FROTA, Lia Gomes da. Oncologia. Atualizações para graduação. São Paulo: Booknando Livros, 2019.

CARREIRO, Allicya Estefany dos S.; FREITAS, Thais Souza de; ARAÚJO, Thamara Maria; SILVA, Sheila da Costa Rodrigues; PAULO, Ana Paula Dantas da Silva. Tratamentos e assistência de enfermagem frente ao câncer de mama. Anais VI CONGREFIP. Campina Grande: Realize Editora, 2017.

CORBELLINI, Bruna; COSTA, Arlete Eli Kunz da; PISSAIA, Luís Felipe. Sistematização da assistência de enfermagem em pacientes com câncer de mama: a atuação do enfermeiro. Research, Society and Development, v. 8, n. 9, p. oI-I4, 2019.

CORREA, Luiz Nilton. Metodologia Científica: Para trabalhos acadêmicos e artigos científicos. II ed. Florianópolis/SC: Do Autor, 2017.

CUSTÓDIO, Ana Carolina. Modelo teórico de cuidado de enfermagem às mulheres em tratamento de câncer de mama na saúde suplementar. Dissertação (mestrado) Universidade Federal de Santa Catarina, Centro de Ciências da Saúde, Programa de PósGraduação em Enfermagem, Florianópolis, 2019.

DERENZO, Neide; COSTA, Maria Antonia Ramos; MELO, Willian Augusto de; COSTA, Cassia Kely Favoretto; FRANCISQUETI, Verônica; BERNUCI, Marcelo Picinin. Conhecimento de mulheres sobre fatores relacionados ao câncer de mama. Revista de Enfermagem UFSM; 7(3): 436-447; 2017.

DIAS, Ricardo Freitas. Metodologia Cientifica - Um guia prático para profissionais da saúde. Petrolina/PE: ScienceeArt, 2017.

FEITOSA, Elizabete Modesto; SÁ, Maria Amélia Pereira de; ANDRADE, Erci Gaspar da Silva; SANTOS, Walquiria Lene dos. Assistência de Enfermagem no rastreamento do câncerde mama. Revista JRG de Estudos Acadêmicos. Ano I, vol. I, n.3 (extra), 27-35, 2018.

FERRARI, Carolina Ferdinatta; ABREU, Edimara Ceolin de; TRIGUEIRO, Tatiane Herreira; SILVA, Marly Bittencourt Gevársio Marton da; KOCHLA, Kátia Antunes; SOUZA, Silvana Regina Rossi Kissula. Orientações de cuidado do enfermeiro para a mulher em tratamento para câncer de mama. Revista de enfermagem UFPE on line, Recife, I2(2):68o-5, fev., 2018. 
GONÇALVES, Juliana Garcia; SIQUEIRA, Alessandra de Sá Earp; ROCHA, Isabel Garcia de Almeida; LIMA, Eduardo Fernandes Felix de; ALVES, Letícia da Silva; SILVA, Beatriz Oliveira da; MENDONÇA, Paulo Eduardo Xavier de; MERHY, Emerson Elias; LAND, Marcelo Gerardin Poirot. Evolução histórica das políticas para o controle do câncer de mama no Brasil. Diversitates International Journal, v. 8, n. I, 2016.

INACIO, Daniela; VENSON, Fernanda Duarte. Cuidados de enfermagem à pessoa com câncer de mama em unidade de internação e ambulatório hospitalar. Trabalho de Conclusão de Curso (Graduação em Enfermagem). Criciúma: Universidade do Extremo Sul Catarinense, 2020.

INSTITUTO NACIONAL DE CÂNCER JOSÉ ALENCAR GOMES DA SILVA INCA. Estatísticas de câncer. Disponível em: https://www.inca.gov.br/numeros-decancer. Acesso em Iomar202I.

LAKATOS, Eva Maria; MARCONI, Marina de Andrade. Metodologia Cientifica. 7.ed. São Paulo: Atlas, 2017.

MELO, Fabiana Barbosa Barreto; MARQUES, Carla Andréia Vilanova; ROSA, Anderson da Silva; FIGUEIREDO, Elisabeth Níglio de; GUTIERREZ, Maria Gaby Rivero de. Ações do enfermeiro na detecção precoce do câncer de mama. Revista Brasileira de Enfermagem, v. 70, p. III9-II28, 2017.

MIGOWSKI, Arn; STEIN, Airton Tetelbom; FERREIRA, Camila Belo Tavares; FERREIRA, Daniele Masterson Tavares Pereira; NADANOVSKY, Paulo. Diretrizes para deteç̧ão precoce do câncer de mama no Brasil. I-Métodos de elaboração. Cadernos de Saúde Pública, v. 34, p. eoor16317, 2018.

MULLER, Elaine Teresinha; PEREIRA, Adriana Dall'Asta; ZAMBERLAN, Claudia; FERREIRA, Carla Lizandra de Lima. Contribuição da enfermagem na reabilitação da mulher com câncer de mama: revisão narrativa. Disciplinarum Scientia - Saúde, v. I9, n. 2, p. 255-265, 2018.

NETO, Jacinto da Costa Silva. Citologia Clínica da Mama. Rio de Janeiro: Thieme Revinter, 2019.

OHL, Isabella Cristina Barduchi; OHL, Rosali Isabel Barduchi; CHAVAGLIA, Suzel Regina Ribeiro; GOLDMAN, Rosely Erlach. Ações públicas para o controle do câncer de mama no Brasil: revisão integrativa. Revista Brasileira de Enfermagem, v. 69, n. 4, p. 793803, 2016.

OLIVEIRA, Murielly Marques de; CORREIA, Alana Barbosa; FERREIRA, Lucelha Correia. Ações do enfermeiro na detecção precoce do câncer de mama em mulheres no Brasil: revisão integrativa. Revista Científica FacMais; II(4): 28-49, 2017. 
PEREIRA, Ana Carolina Almeida; OLIVEIRA, Déborah Viana; ANDRADE, Smalyanna Sgren da Costa. Sistematização da assistência de enfermagem e o câncer de mama entre mulheres. Revista de Ciências da Saúde Nova Esperança; i6(I): 39-47, 2018.

REZENDE, Laura; CAMPANHOLI, Larissa Louise; TESSARO, Alessandra. Manual de Condutas e Práticas Fisioterapêuticas no Câncer de Mama da ABFO. Rio de Janeiro: Thieme Revinter, 2018.

RODRIGUES, Josiane Ramos Garcia; SALUN, Ariana Aparecida Lins Aleksandrovic; OLIVEIRA, Vanessa Aparecida Sanches Campassi de; LIMA, Priscila Bocchile de; NUNES, Maria Renata. Importância do enfermeiro para o controle do câncer de mama: revisão narrativa. Revista Eletrônica Acervo Saúde, n. 55, p. e3668, 23 jul. 2020.

SANTOS, Glenda Dias dos; CHUBACI, Rosa Yuka Sato. O conhecimento sobre o câncer de mama e a mamografia das mulheres idosas frequentadoras de centros de convivência em São Paulo (SP, Brasil). Ciência \& Saúde Coletiva, I6(5):2533-2540, 2017.

SEVERINO, Antonio Joaquim. Metodologia do trabalho científico. 2.ed. São Paulo: Cortez, 2017.

SILVA, Raimunda Magalhães da; FERNANDES, Ana Fátima Carvalho; PINHEIRO, Cleoneide Paulo Oliveira. Câncer de mama: políticas e práticas na rede de atenção à saúde. Curitiba: Editora CRV, 2020.

SILVEIRA, Cristiane Martins Bolonha; GONÇALVES, Eduarda Frare; FIDELIS, Fabiana Aparecida Monção; SANTANA, Isadora Ribeiro; SARRACENI, Jovira Maria; FERRARI, Luiz Felipe da Silva. Atuação da equipe de enfermagem frente a prevenção e diagnóstico precoce do câncer de mama: uma revisão integrativa. Brazilian Journal of Development, v. 7, n. 7, p. 72233-72248, 2021.

SMELTZER, Suzanne C.; BARE, Brenda G. (orgs), Brunner \& Suddarth, tratado de enfermagem médico-cirúrgica. Trad. José Eduardo Ferreira de Figueiredo. Rio de Janeiro: Guanabara Koogan, 2017.

SOUSA, Luís Manuel Mota de; VIEIRA, Cristina Maria Alves Marques; SEVERINO, Sandy Silva Pedro; ANTUNES, Ana Vanessa Antunes. A metodologia de revisão integrativa da literatura em enfermagem. Revista Investigação em Enfermagem. n. 2I Série 2. $17-26,2017$.

SOUSA, Samara Maria Moura Teixeira; CARVALHO, Maria das Graças Freire de Medeiros; SANTOS JÚNIOR, Luiz Ayrton; MARIANO, Sarah Borges Carolina Marianoi. Acesso ao tratamento da mulher com câncer de mama. Saúde em Debate, v. 43, p. 727-741, 2019. 
SOUZA, Tábata de Cavatá; MONTEIRO, Daiane da Rosa; TREVISAN, Bibiana Fernandes; MALLMANN, Francielly Haygertt. Atuação da enfermagem no cuidado a pacientes com câncer de mama: revisão integrativa. Research, Society and Development, v. 9, n. I2, p. el4391210939-eI4391210939, 2020.

TEIXEIRA, Luiz Antonio; ARAÚJO NETO, Luiz Alves. Câncer de mama no Brasil: medicina e saúde pública no século XX. Saúde e Sociedade, v. 29, p. er80753, 2020.

TEIXEIRA, Michele de Souza; GOLDMAN, Rosely Erlach; GONÇALVES, ValterliConceição Sanches; GUTIÉRREZ, Maria Gaby Rivero de; FIGUEIREDO, Elisabeth Níglio de. Atuação do enfermeiro da Atenção Primária no controle do câncer de mama. Acta Paulista de Enfermagem, v. 30, n. I, p. I-7, 2017.

ZUCCA-MATTHES, Gustavo. Câncer de Mama - Uma filosofia de tratamento Breast Unit Barretos-Bub. Rio de Janeiro: Thieme Revinter, 2018. 